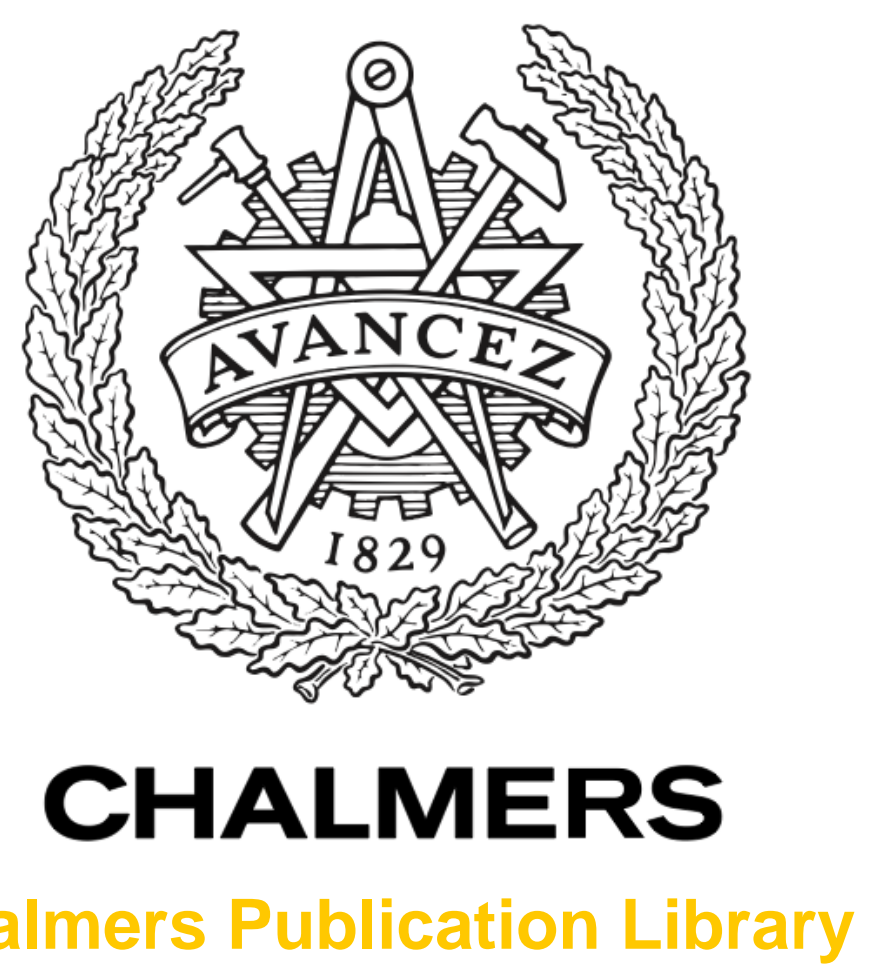

\title{
Resource Allocation for Flexible-Grid Optical Networks With Nonlinear Channel Model
}

This document has been downloaded from Chalmers Publication Library (CPL). It is the author's version of a work that was accepted for publication in:

Journal of Optical Communications and Networking (ISSN: 1943-0620)

Citation for the published paper:

Yan, L. ; Agrell, E. ; Wymeersch, H. et al. (2015) "Resource Allocation for Flexible-Grid Optical Networks With Nonlinear Channel Model". Journal of Optical Communications and Networking, vol. 7(11), pp. B101-B108.

Downloaded from: http://publications.lib.chalmers.se/publication/224824

Notice: Changes introduced as a result of publishing processes such as copy-editing and formatting may not be reflected in this document. For a definitive version of this work, please refer to the published source. Please note that access to the published version might require a subscription. 


\title{
Resource Allocation for Flexible-Grid Optical Network with Nonlinear Channel Model
}

\author{
Li Yan, Student Member, IEEE, Erik Agrell, Senior Member, IEEE, Henk Wymeersch, Member, IEEE, and \\ Maïté Brandt-Pearce, Senior Member, IEEE
}

(Invited Paper)

\begin{abstract}
In this paper, we study the joint resource allocation in flexible-grid networks based on a nonlinear physical layer impairment model. An optimization problem is formulated to assign resources and guarantee the signal quality for every channel. Compared with the resource allocation in fixed-grid wavelength-division multiplexing scenario, our method achieves significant bandwidth reduction and transmission distance extension in flexible-grid networks. The maximum spectrum usage is shown to be insensitive to the ordering of channels. We also analyze the relation between modulation formats and transmission distance based on the results of the proposed method. Finally, we demonstrate the performance and scalability of the proposed algorithm in ring networks.
\end{abstract}

Index Terms-Resource allocation, Gaussian noise model, network optimization, optical fiber communications.

\section{INTRODUCTION}

$\mathbf{T}$ RADITIONAL optical networks are based on fixed grids, i.e., all the channels should be confined to $50 \mathrm{GHz}$ frequency grids regardless of their transmission distances or data rates [1]. However, the traffic demands become more diverse and heterogeneous [2], therefore it is impossible to find a uniform grid size that can satisfy all different traffic demands.

Flexible-grid optical networks are a promising solution to the Internet capacity challenge by allocating resources adaptively according to the communication demands [2], [3]. Consequently, various routes, modulations, bandwidths, and power spectral densities (PSDs) can coexist in the networks, resulting in more complicated and significant nonlinear interference (NLI) between channels sharing the same fiber link. This problem is partly tackled by adapting the modulation formats according to light path length [3], where the routes, modulation level, and spectrum assignment (RMLSA) problem is formulated. Several variants of RMLSA and an array of heuristic algorithms have been studied [4]. Most of these methods guarantee quality of transmission (QoT) through over-provisioning of resources [1], [5], which requires large margins for most of the channels to operate error free in the worst case, and therefore causes inefficient resource utilization.

Parts of this paper have been presented at the Optical Fiber Communication Conference (OFC), Los Angeles, CA, Mar. 2015. The research was funded by the Swedish Research Council (VR) under grant no. 2012-5280. L. Yan, E. Agrell, and H. Wymeersch are with Chalmers University of Technology, SE-41296 Gothenburg, Sweden (lyaa@chalmers.se). M. Brandt-Pearce is with University of Virginia, Charlottesville, 22904 Virginia, USA.
Recently, an analytical physical layer impairment model, the Gaussian noise (GN) model [6]-[8] has been proposed, which combines reasonable accuracy and low computational complexity. Based on this model, optimization of fixed-grid wavelength-division multiplexing (WDM) networks with static traffic demands have been done both at link level [9] and meshed network level [10] to improve the overall network throughput by allowing the transmitter launch powers and modulation formats to be different for each channels, and optimizing these variables jointly. In the WDM dynamic traffic scenario, congestion-aware routing [11] was also studied with a nonlinear signal distortion model, and variable coding-rate and modulation formats to increase the network capacity greatly.

Resource allocations in flexible-grid networks have also been proposed [12]-[14], where channel power or channel spacings are separately optimized. However, such separate optimization cannot fully harness the advantages of the flexiblegrid network, and thus only yields sub-optimal solutions. Joint resource allocation in flexible-grid network has been investigated on link level [15], with carrier frequencies, modulation formats, and PSD optimized jointly based on GN model. Similar algorithms have been proposed in dynamic flexible-grid network [16] to include nonlinear impairment through a power-optimized reach constraint. These algorithms consider the same PSD for all the channels, while individually optimized transmitter launch power has already been demonstrated beneficial in improving the network throughput [17]. In flexible-grid networks, the PSD per channel has been jointly optimized together with modulation formats and carrier frequencies based on the GN model [18].

In this paper, we use the GN model [8] to estimate the NLI. Resources are optimized with the primary goal of minimizing the maximum bandwidth usage, and a secondary goal of maximizing the signal-to-noise ratio (SNR) margins of all the channels. We extend our previous work [18] by analyzing the relation between modulation formats and transmission distances in a simple tandem network. The proposed algorithm is also applied to realistic networks to demonstrate its performance and scalability. Hence, this work can be seen an extension of the link level resource allocation algorithm [15] to more complex topologies with optimized PSD per channel as well.

The remainder of the paper is organized as follows. In Section II, we describe the problem to be solved in this paper. We present the channel model and the nonlinear SNR 
constraint in Section III. In Section IV, we formulate an optimization problem to allocate resources jointly. As examples, the proposed method is applied to both a simple tandem network and ring networks with various numbers of nodes in Section V. Concluding remarks are found in Section VI.

\section{Problem Statement}

In this paper, we consider a transparent optical network as a graph $(V, E)$, where $V$ is the set of nodes and $E$ is the set of links. The spectrum on fiber links is assumed gridless. Each link has two fibers with opposite directions and multiple spans. $D$ is used to denote the set of channels that carry demanded requests, and $D_{l}$ is the set of channels on link $l .|D|$ represents the number of elements in set $D$, i.e., the total number of channels. The available modulation formats in the network and their spectral efficiencies $c$ and required minimum SNR thresholds $\mathrm{SNR}_{\mathrm{th}}(c)$ under certain pre-forward error correction (FEC) bit-error-rate $(\mathrm{BER})$ requirement $\left(4 \times 10^{-3}\right.$ in this paper) are listed in Table I. Since the rows in Table I have different spectral efficiencies, we will use $c$ to denote different modulation formats. A static traffic scenario is considered, where $R_{i}$ (in Gbps) denotes the data rate request $i$, which is served by a channel $i \in D$. For the same source $s$ and destination $d$, there can be multiple traffic demands, each of which is carried by a different channel.

Assuming Nyquist spectrum shaping, channel $i$ with data rate $R_{i}$ and modulation format $c_{i}$ has bandwidth $\Delta f_{i}=R_{i} / c_{i}$. Other resources allocated to channel $i$ include the PSD $G_{i}$, the carrier frequency $f_{i}$, and a route consisting of a sequence of links connecting the source $s_{i}$ and destination $d_{i}$. Fig. 1 illustrates two possible spectra allocated to three channels and various parameters defined above. The number of spans transversed by the route allocated to channel $i$ is denoted as $N_{i}^{\mathrm{s}}$, and the number of spans shared by the routes of channel $i$ and $j$ is denoted as $N_{i j}^{\mathrm{s}}$. Based on the resources allocated to all the channels, we are able to calculated their QoTs in terms of SNR by using the GN model described in Section III.

The primary goal of the resource allocation problem considered in this paper is to minimize the bandwidth usage of the network, while guaranteeing the QoT of all the traffic demands by providing satisfactory SNRs. And the secondary goal is to maximize the SNR margins of all the channels. In general, such a problem can be decomposed into two subproblems: i) the routing and spectrum ordering problem, where the routing of each channel, and the ordering of channels on each link

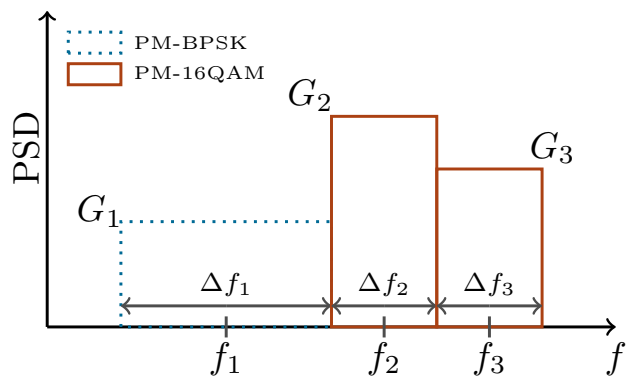

Table I

THE AVAILABLE MODULATION FORMATS, THEIR SPECTRAL EFFICIENCIES $c$, AND LINEAR SCALE SNR THRESHOLDS SNR $\mathrm{SH}_{\mathrm{TH}}(c)$ TO ACHIEVE A PRE-FEC BER OF $4 \times 10^{-3}$.

\begin{tabular}{lcc}
\hline Modulation Format & $c$ (bit/s/Hz) & SNR $_{\text {th }}(c)$ \\
\hline PM-BPSK & 2 & 3.52 \\
PM-QPSK & 4 & 7.03 \\
PM-8QAM & 6 & 17.59 \\
PM-16QAM & 8 & 32.60 \\
PM-32QAM & 10 & 64.91 \\
PM-64QAM & 12 & 127.51 \\
\hline
\end{tabular}

are defined, and ii) allocation of the remaining resources. Different from the spectrum assignment, spectrum ordering only specifies the relative allocation of channels, e.g., channel $i$ is at the right of $j$, but the carrier frequencies and bandwidths of $i$ and $j$ are not concerned. It is generally too hard to solve these two subproblems jointly. This is because without the final routing solution we do not know how the channels interact with each other, and thus we are unable to calculate the NLI or allocate bandwidths and PSDs [10]; while without the exact NLI estimations, it is hard to find the best routing solution. Usually the search for close-to-optimal solution involves iterations between these two subproblems [17]. In this work, we mainly focus on the second subproblem, and assume the routing and spectrum ordering are predetermined [3].

Based on the description above, our resource allocation algorithm takes as input parameters the network topology $(V, E)$, the routing and spectrum ordering solutions, and traffic demands $R$ for all the channels in $D$. The output parameters of the algorithm consist of modulation formats $c$, carrier frequencies $f$, and PSDs $G$ allocated to every channel.

\section{GN MODEL AND SNR CONSTRAINT}

The GN model [6]-[8] considers the NLI between channels caused by the Kerr effect as additive Gaussian noise, and combines incoherently with the additive spontaneous emission (ASE) noise due to fiber amplifiers. Thus, it is possible to approximately calculate the NLI and the SNR for all channels in a network. The model holds under the following assumptions [8]: (i) a coherent polarization-multiplexed system without inline compensation for chromatic dispersion; (ii) for each channel, the transmitted signal PSD is equal in both polarizations; (iii) each channel spectrum is rectangular and does not overlap with neighboring channels; (iv) the NLIs

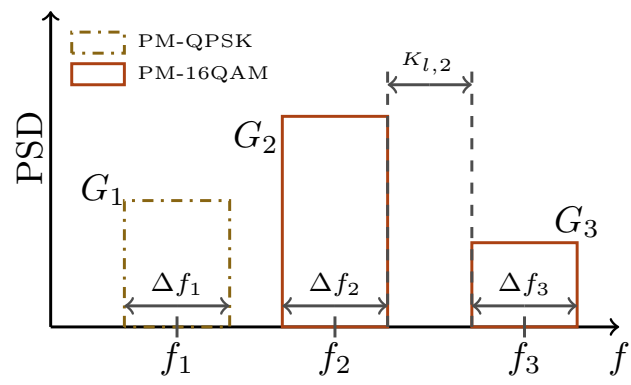

Figure 1. An example of resources allocated to three channels. $K_{l, 2}$ denotes the frequency interval between channels 2 and 3 on this link $l$. 
generated in different fiber spans sum incoherently over the whole link; (v) the loss of each fiber span is compensated by an EDFA at the end of the span; (vi) all spans are long enough to have the same effective length; (vii) multi-channel interference [6], [8] is neglected.

The NLI PSD (in $\mu \mathrm{W} / \mathrm{Hz}$ ) per polarization for channel $i \in$ $D$ can then be expressed as [8]

$$
\begin{aligned}
G_{i}^{\mathrm{SCI}} & =\frac{3 \gamma^{2}}{\alpha^{2}} F_{i i}^{2} G_{i}^{3} N_{i}^{\mathrm{s}} \\
G_{i}^{\mathrm{XCI}} & =\frac{6 \gamma^{2}}{\alpha^{2}} G_{i} \sum_{\substack{j \in D \\
j \neq i}} F_{i j}^{2} G_{j}^{2} N_{i j}^{\mathrm{s}},
\end{aligned}
$$

where $G_{i}^{\mathrm{SCI}}$ is the PSD of self-channel interference (SCI), $G_{i}^{\mathrm{XCI}}$ is the PSD of cross-channel interference (XCI), $\gamma$ is the nonlinear parameter, and $\alpha$ is the fiber power attenuation. $F_{i j}^{2}$ for $i, j \in D$ is the NLI efficiency function representing the NLI effects [8]

$$
\begin{aligned}
F_{i j}^{2}= & \frac{2}{\xi}\left\{\operatorname{ImLi}_{2}\left[\sqrt{-1} \frac{\Delta f_{i}}{2}\left(f_{i}-f_{j}+\frac{\Delta f_{j}}{2}\right) \xi\right]\right. \\
& \left.+\operatorname{Im~Li}_{2}\left[\sqrt{-1} \frac{\Delta f_{i}}{2}\left(f_{j}-f_{i}+\frac{\Delta f_{j}}{2}\right) \xi\right]\right\},
\end{aligned}
$$

with

$$
\xi=\frac{4 \pi^{2}\left|\beta_{2}\right|}{\alpha}
$$

Here $\mathrm{Li}_{2}$ is the dilog function [8], and $\beta_{2}$ is group velocity dispersion.

Note that in (2), the computation involves the dilog function, which is fairly complex. In numerical calculations, functions with simpler forms and less complexity are preferable. Using the asymptotic expansion of dilog function, the NLI efficiency function $F_{i j}^{2}$ for $i, j \in D$ can be approximated by the hyperbolic arcsin function [19] if $i=j$, and the logarithm function [8] if $i \neq j$

$$
\begin{aligned}
& F_{i i}^{2} \approx \frac{\alpha}{2 \pi\left|\beta_{2}\right|} \operatorname{arcsinh}\left(\frac{\pi^{2}\left|\beta_{2}\right|}{2 \alpha} \Delta f_{i}^{2}\right), \\
& F_{i j}^{2} \approx \frac{\alpha}{4 \pi\left|\beta_{2}\right|} \log \left|\frac{\left|f_{i}-f_{j}\right|+\Delta f_{j} / 2}{\left|f_{i}-f_{j}\right|-\Delta f_{j} / 2}\right|, i \neq j .
\end{aligned}
$$

The hyperbolic arcsin function is preferred over the logarithm for the SCI coefficient $F_{i i}^{2}$ as it approximates the dilog function better when $\pi^{2}\left|\beta_{2}\right| \Delta f_{i}^{2} / 2 \alpha \ll 1$, in which case the logarithm function tends to negative infinity. On the contrary, the argument of the XCI coefficient $F_{i j}^{2}$ in (4) is always greater than 1 , and thus the logarithm function gives acceptable approximation error.

In addition to the NLI, each EDFA adds white Gaussian noise with PSD

$$
G_{i}^{\mathrm{ASE}}=\left(e^{\alpha L}-1\right) h \nu n_{\mathrm{sp}} N_{i}^{\mathrm{s}},
$$

where $L$ is the fiber length per span, $n_{\mathrm{sp}}$ is the spontaneous emission factor, $\nu$ is the light frequency, and $h$ is Planck's constant. Combining the linear and nonlinear noises, the SNR for channel $i \in D$ can be expressed as

$$
\mathrm{SNR}_{i}=\frac{G_{i}}{G_{i}^{\mathrm{ASE}}+G_{i}^{\mathrm{XCI}}} .
$$

The SCI is neglected because we assume that the SCI noise can be fully mitigated by suitable receiver design [9], [20].

In order to obtain satisfactory QoT for each channel, a certain SNR requirement should be met. This can be written as

$$
\operatorname{SNR}_{i}(\mathbf{c}, \mathbf{f}, \mathbf{G}) \geq \operatorname{SNR}_{\mathrm{th}}\left(c_{i}\right), \quad \forall i \in D .
$$

Here $\operatorname{SNR}_{i}(\mathbf{c}, \mathbf{f}, \mathbf{G})$ is the $\mathrm{SNR}$ of channel $i$ calculated according to (6) as a function of the modulation formats, carrier frequencies, and PSDs allocated to all the channels in the network. $\mathbf{c}=\left(c_{1}, \ldots, c_{|D|}\right)^{\mathrm{T}}$ is the vector of spectral efficiencies, $\mathbf{f}=\left(f_{1}, \ldots, f_{|D|}\right)^{\mathrm{T}}$ is the vector of carrier frequencies, and $\mathbf{G}=\left(G_{1}, \ldots, G_{|D|}\right)$ is the vector of PSDs.

\section{OPTIMIZATION FORMULATION}

Using the SNR constraints in (7) and the objectives described in Section II, the resource allocation can be formulated as

$$
\begin{array}{llr}
\underset{\mathbf{c}, \mathbf{f}, \mathbf{G}, u, \mathbf{t}, t_{\min }}{\operatorname{minimize}} & \theta_{1} u+\theta_{2} \sum_{i \in D} \frac{R_{i}}{c_{i}} G_{i}+\theta_{3} t_{\text {min }}+\theta_{4} \sum_{i \in D} t_{i} \\
\text { subject to } & t_{i} \operatorname{SNR}_{i}(\mathbf{c}, \mathbf{f}, \mathbf{G}) \geq \operatorname{SNR}_{\mathbf{t h}}\left(c_{i}\right), & \forall i \in D \\
& \mathbf{K}_{l}(\mathbf{c}, \mathbf{f}) \leq 0, & \forall l \in E \\
& u \geq \frac{R_{i}}{2 c_{i}}+f_{i}, & \forall i \in D \\
& t_{\text {min }} \geq t_{i}, & \forall i \in D,
\end{array}
$$

where $u$ is the upper bound of the occupied frequency, $t_{i}$ is the reciprocal of the SNR margin in linear scale for channel $i$, and $t_{\min }$ represents the inverse of the minimum SNR margin.

For every link $l$, all channels using it should not overlap with any other channels on the same link. This nonoverlapping requirement is modeled by an element-wise nonpositive constraint on the vector $\mathbf{K}_{l}(\mathbf{c}, \mathbf{f})$ where $\mathbf{K}_{l}(\mathbf{c}, \mathbf{f})=$ $\left(K_{l, 1}(\mathbf{c}, \mathbf{f}), \ldots, K_{l,\left|D_{l}\right|-1}(\mathbf{c}, \mathbf{f})\right)^{\mathrm{T}}$ for $l \in E$, and

$$
K_{l, j}(\mathbf{c}, \mathbf{f})=\frac{1}{2}\left(\frac{R_{i_{j}^{l}}}{c_{i_{j}^{l}}}+\frac{R_{i_{j+1}^{l}}}{c_{i_{j+1}^{l}}}\right)+f_{i_{j}^{l}}-f_{i_{j+1}^{l}} .
$$

Here $i_{j}^{l}$ is the $j^{\text {th }}$ channel on link $l$, and as illustrated in Fig. $1, K_{l, j}(\mathbf{c}, \mathbf{f})$ denotes the spectrum interval between two neighboring channels.

The formulation in (8) is flexible enough to have multiple objectives associated with different weights: i) minimizing the maximum bandwidth usage on all the links, so that the network can have resources for more traffic demands; ii) minimizing the total optical power in the fiber to reduce nonlinearity in the fiber; and iii) maximizing the minimum and total SNR margins of all the channels to improve robustness and capacity of the network. These objectives are traded off by the weighting factors $\theta_{1}, \theta_{2}, \theta_{3}$, and $\theta_{4}$, respectively.

The formulation (8) is a mixed integer nonlinear programming problem (MINLP). Its solution is obtained by outer approximating the nonlinear constraints and applying branchand-bound, which is implemented in BONMIN [21], a generic MINLP solver. The solver works by recursively dividing the solution space of the original problem into smaller subsets, on each of which a subproblem is formulated. A convex relaxation 
or outer approximation [22] of each subproblem is solved to provide a lower bound of solution for that subset, while an upper bound is obtained by a feasible solution. The gap between the highest lower bound and the lowest upper bound among all the subproblems is called optimality gap, which indicates the convergence and can be used as a termination criterion.

Note that in (8) we assume a gridless spectrum. To extend the result to finite-grid networks, we can either round the carrier frequencies to the next multiple of the grid granularity [23], or solve the problem again with carrier frequencies being constrained to integer multiples of the grid size. While the first method may lead to infeasible solutions with unsatisfactory channel SNRs, the second method increases the complexity of the problem considerably. Both methods will result in increased bandwidth utilization because more constraints are added to (8) and the solution space shrinks. However, we will show in Section V-B the increase of bandwidth usage is marginal with modern switching technology that allows a spectrum resolution down to $3.125 \mathrm{GHz}$ [2].

The number of variables used in the above MINLP formulation is $4|D|+2$, and the number of constraints depends on the routing solution. In particular, constraint $\mathbf{K}_{l}(\mathbf{c}, \mathbf{f}) \leq 0$ needs to be employed for channel $i$ only if its route uses link $l$. In the worst case, $(|D|-1) \times|E|$ non-overlapping constraints would be required. The rest of the formulation uses $3|D|$ constraints. In general, MINLP is an NP-hard [22] problem, and thus is impossible to be solved efficiently for large $|D|$ and $|E|$. If routing and spectrum ordering are included, the formulation would be even more complex. The complexity issue is the reason why we decompose the resource allocation into subproblems.

\section{NumERICAL RESUlts}

To evaluate the performance of the proposed optimization formulation, we compare with the fixed-grid network planning approach [9], considering the same tandem network shown in Fig. 2 as well as ring networks with various numbers of nodes. The optimizations are solved on a $3.4 \mathrm{GHz}$ Quad-Core computer with 8 GB RAM memory. The problem is terminated if the relative optimality gap is less than $1 \%$ or the running time exceeds 2 hours.

\section{A. Three-Node Chain Network}

For the tandem network, the set of nodes is $D=\{\mathrm{A}, \mathrm{B}, \mathrm{C}\}$, the set of links is $E=\{\mathrm{AB}, \mathrm{BC}\}$. The benchmark [9] uses a fixed-grid WDM-based nonlinear PLI model, which is generalized by the GN model described in Section III. The system parameters are set as $\alpha=0.22 \mathrm{~dB} \cdot \mathrm{km}^{-1}$,

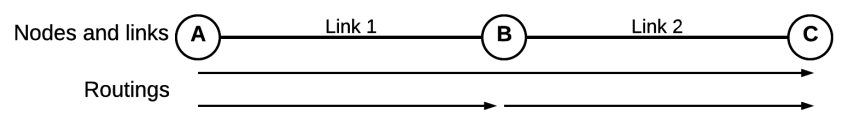

Figure 2. The tandem network. The lengths of the two links are equal. Traffic flows from $\mathrm{A}$ to $\mathrm{B}, \mathrm{A}$ to $\mathrm{C}$, and $\mathrm{B}$ to $\mathrm{C}$.

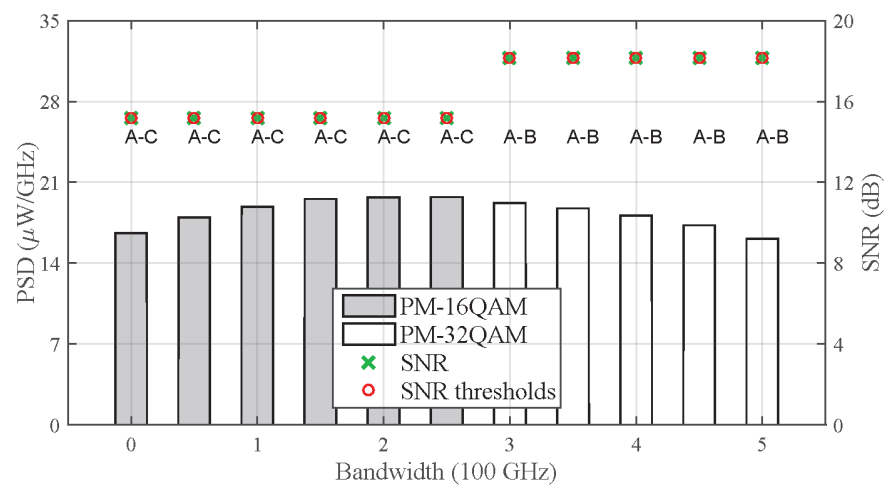

Figure 3. Allocated spectrum for benchmark on link 1 where the traffic demands are $200 \mathrm{Gbps}$ for each of the $6 \mathrm{~A}-\mathrm{C}$ channels, and $250 \mathrm{Gbps}$ for each of the 5 A-B channels. The SNRs are equal to the SNR thresholds for all the channels. The SNR values (crosses and circles) refer to the right-hand side.

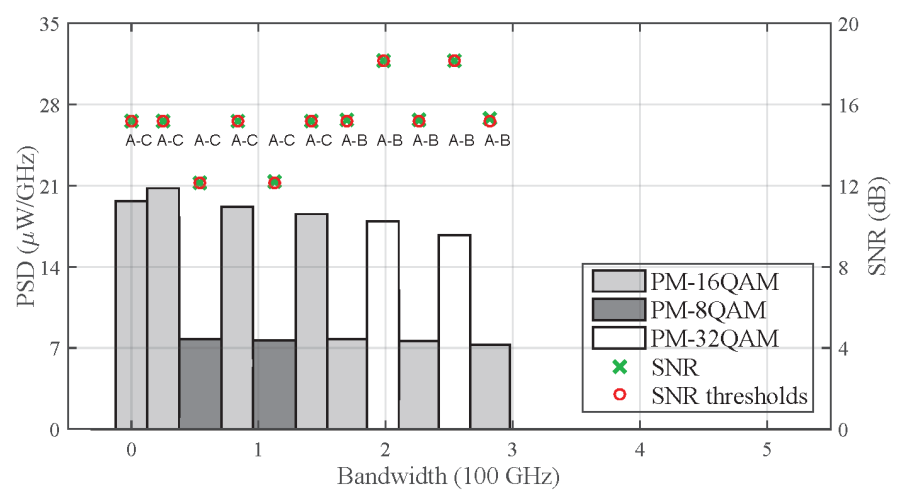

Figure 4. Allocated spectrum for the proposed method on link 1, with the same traffic as in Fig. 3. The SNRs are equal to the SNR thresholds for all the channels.

$\gamma=1.3(\mathrm{~W} \cdot \mathrm{km})^{-1}, \beta_{2}=-2.13 \mathrm{~s}^{2} \cdot \mathrm{m}^{-1}, L=100 \mathrm{~km}$, $n_{\mathrm{sp}}=1.58, \nu=193.55 \mathrm{THz}$.

For the proposed method, the relative values of the weights in (8) are set to $\theta_{1}=1, \theta_{2}=0, \theta_{3}=10^{-3}$, and $\theta_{4}=10^{-3}$, so the optimization prioritizes the bandwidth minimization. For solutions achieving the same minimum spectrum usage, PSDs are adjusted to obtain the maximum total SNR margins. The benchmark algorithm is a special case of (8) by fixing the carrier frequencies of all channels to the WDM grids, and setting the weights as $\theta_{1}=0, \theta_{2}=0, \theta_{3}=1$, and $\theta_{4}=0$. Here $\theta_{1}$ is set to 0 because it is meaningless to minimize spectrum usage in fixed-grid network; $\theta_{2}$ is set to 0 to reproduce exactly the same results as the benchmark [9]. In the proposed method the values of $\theta_{3}$ and $\theta_{4}$ are much smaller than $\theta_{1}$, and thus have no effect on spectrum minimization. The running times of all the proposed and the benchmark algorithms are less than 1 minute for each instance.

First, we compare the total bandwidths used by both schemes. The number of spans of both links is 6 , and there are 5 channels between A-B and B-C, 6 channels between A-C. In [9], by optimizing the channel powers and modulation formats, 2.45 Tbps throughput on each link is achieved using $575 \mathrm{GHz}$ of spectrum. Fig. 3 reproduces the 


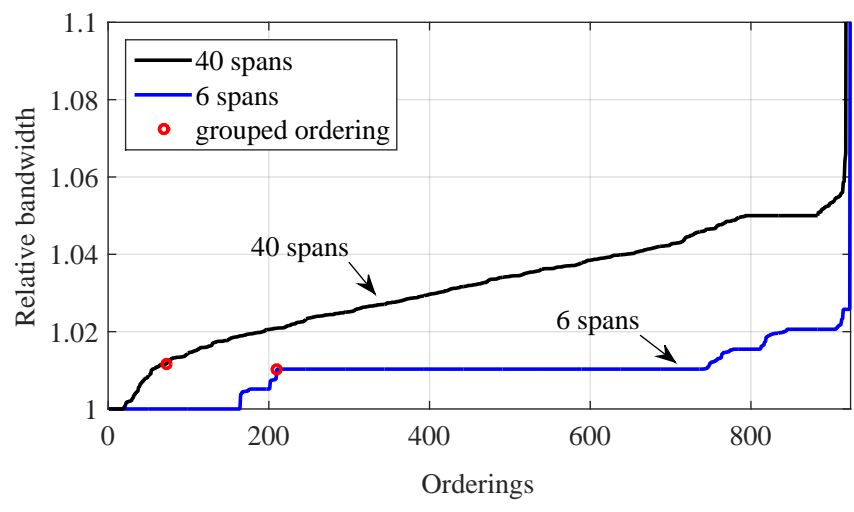

Figure 5. Results of optimized bandwidths for all orderings. Results are sorted and normalized to the minimum bandwidth. There are 18 channels in the whole network, 6 for each source-destination pair, and $400 \mathrm{Gbps}$ on each channel.

benchmark, where 6 A-C channels use PM-16QAM with data rates of $200 \mathrm{Gbps}$ and 5 A-B channels use PM-32QAM with $250 \mathrm{Gbps}$. The resulting power and SNRs are well in line with [9, Fig. 2(c)]. When the fixed grid is relaxed to flexible grid, given the same channel configurations and traffic demands, the bandwidth usage is reduced to $325 \mathrm{GHz}$ by solving (8). This $43 \%$ bandwidth saving comes from removing the guardband. By selecting modulation formats adaptively, the channels are more robust to NLI, and thus require less guardband for guaranteeing the QoT. Note that a guardband can be included in (8) and be any preset value according to the wavelength selective switch, and the algorithm will give the corresponding solution. The allocated PSD and modulation formats are not as with regular as the benchmark algorithm. The reason is that we have a larger solution space to search for the joint optimum. Note that with the proposed method, more lower-order modulation formats are employed than with the benchmark, which results in larger bandwidth usage for individual channels. This is nevertheless beneficial for the system as a whole, since reduced modulation order means higher robustness to NLI from neighboring channels.

Second, we investigate the impact of channel ordering on the bandwidth utilization. For the network in Fig. 2 with 6 channels for each source-destination pair, Fig. 5 shows the optimized bandwidths of all orderings, normalized to the minimum bandwidth. Different link lengths are considered as well. We observe that in most cases, the variations are less than $6 \%$, indicating that, contrary to [9], our proposed algorithm is relatively insensitive to channel orderings. As the number of spans grows longer, the variation slightly increases, and a small number of poor orderings (i.e., requiring relatively larger bandwidth) appear. However, as marked by red circles in Fig. 5, such poor orderings can be avoided by grouping channels with the same source and destination together. Actually, the grouped orderings are within $1.5 \%$ of the optimal spectrum allocation in terms of bandwidth usage. The main reason of this ordering insensitivity comes from adding carrier frequencies as a new degree of freedom, which can compensate the extra NLI due to imperfect ordering.

To further understand the impact of the link length, Fig. 6 shows the optimized bandwidth as a function of the number of spans in each link (both links the same) for different data rates per channel. Each source-destination pair has 6 channels, whose spectral orderings are grouped together. The dashed part of the blue lines are the distances for which the benchmark is feasible. As indicated by the red horizontal line, the benchmark uses $575 \mathrm{GHz}$ bandwidth regardless of the data rate and distance. For shorter distances, our algorithm requires less spectrum than the benchmark, while for longer distances, our algorithm still allows data transmission with reasonable spectrum. The reduced bandwidth usage is achieved through removing unnecessary guardband between channels (as shown in Fig. 4), while the extended transmission distance is obtained through using lower-order modulation formats and separate channels further apart. The small peak at 34 spans (marked by red ellipse in Fig. 6) is due to the unoptimized ordering. The allocated spectra of the crosses in Fig. 6 are shown in Fig. 7. In contrast to Fig. 4, our optimization algorithm assigns different guardbands between channels to guarantee satisfactory SNRs. And the resulted SNRs are the same as the SNR thresholds for all the channels.

Contrary to the traditional transmission reach method which assigns a specific modulation format to given data rate and light path length (as illustrated in Fig. 8(a)), Fig. 7 shows that the optimal assignment is a combination of different modulation formats. To study how the link length and data rate affect the choice of modulations, we plotted the ratio of contributions of available modulations in Fig. 8. As is shown, the combination of a range of modulation formats is more favorable for efficient bandwidth usage. As the distance grows, the contribution of lower-order modulations gradually increases. The fluctuations in Fig. 8(b) are again due to the grouped ordering. Note that these fluctuations do not necessarily result in bandwidth usage changes in Fig. 6 due to the flexible carrier frequency assignment. In addition, the ratio of contributions is relatively insensitive to the data rate variations. The observations from Fig. 8 can help us rule out unsuitable solutions in the search space of (8), and thus accelerate the optimization process.

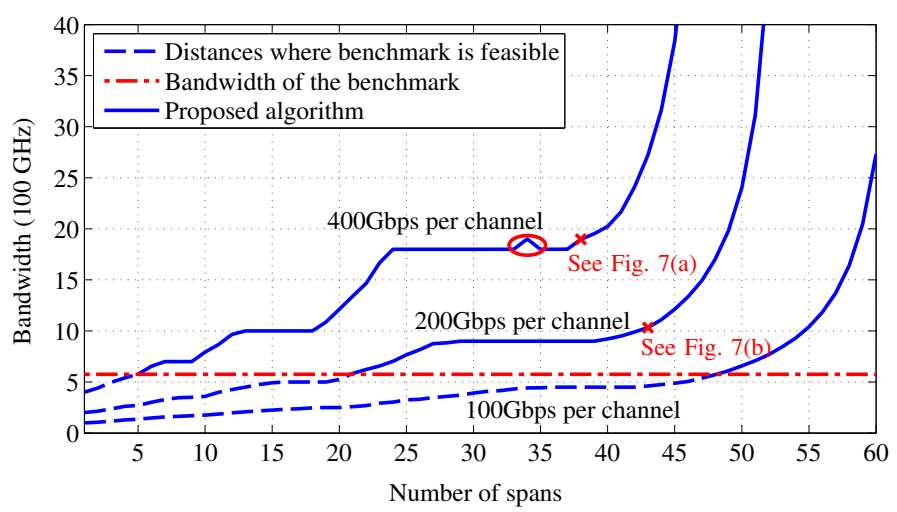

Figure 6. Results for the optimized bandwidth as a function of the length of each link and the data rate per channel. 6 channels with the same sourcedestination pair are grouped together. The blue dashed lines represent the distances where the benchmark is feasible. 


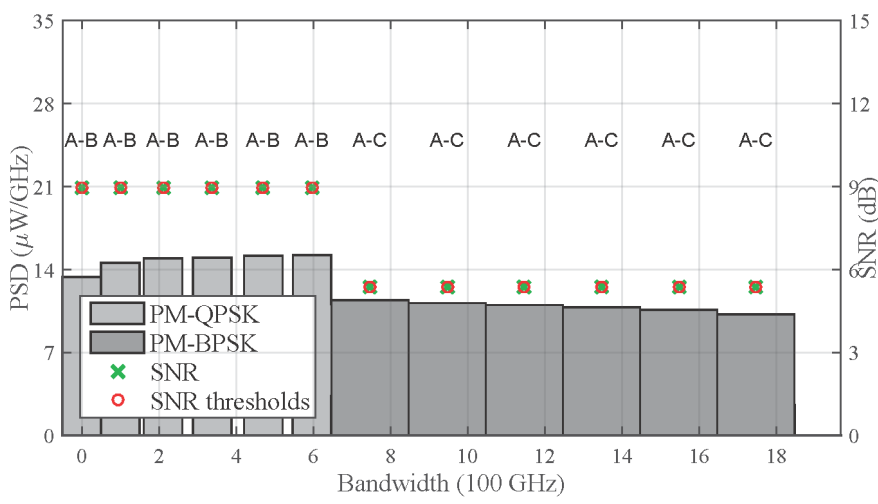

(a)

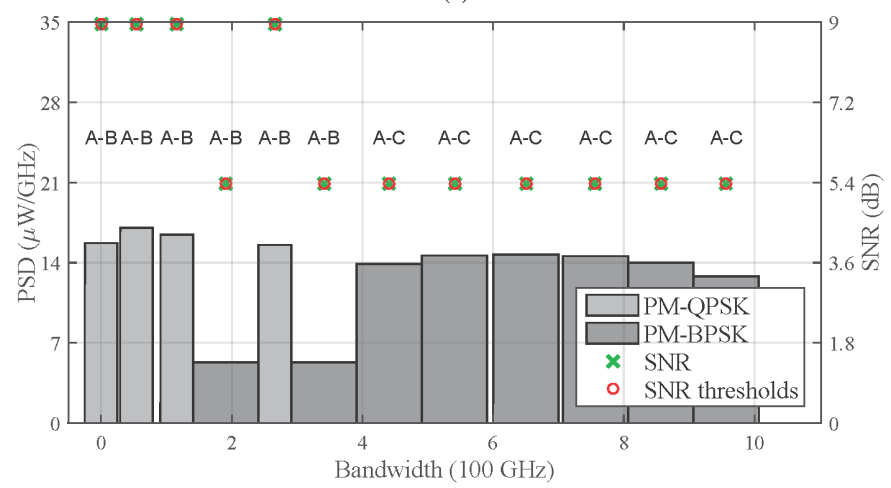

(b)

Figure 7. Allocated spectra on link 1 by the proposed method. The SNRs are equal to the SNR thresholds for all channels. (a) 38 spans for each link and 400 Gbps for each channel (b) 43 spans for each link and 200 Gbps for each channel.

\section{B. Ring Networks}

After optimizing the tandem network, we investigate the performance and scalability of the proposed algorithm in more complex networks. Ring networks with various numbers of nodes rather than meshed networks are chosen for this purpose because one traffic demand can only have two possible routes, i.e., clockwise and counter-clockwise. As a result, we can easily obtain close-to-optimal routing and spectral ordering solution with the simulated annealing meta-heuristic [3], which facilitates the comparison of algorithms studied in this paper. On the contrary, meshed networks with more complex topologies usually involve harder routing and spectral assignment problems, and thus result in poor performance of the heuristics. The inefficient routing and spectral ordering, in turn, will limit the performance of following resource allocation algorithms.

The set of nodes in ring networks is $D=\left\{A_{1}, \ldots, A_{|D|}\right\}$, the set of links is $E=\left\{A_{1} A_{2}, \ldots, A_{|D|} A_{1}\right\}$. Each link consists of two fiber spans. There is one traffic demand between each source-destination pair, each of which has a data rate of $100 \mathrm{Gbps}$. All the system parameters and optimization weights are the same as those in V-A.

The bandwidth usages of the proposed and benchmark algorithms are shown in Figure 9. The spectra allocated by both algorithms grow quadratically as the number of nodes increases. However, the proposed algorithm always uses less spectrum resources than the benchmark. The relative bandwidth reductions are around $40 \%$ regardless of the number of

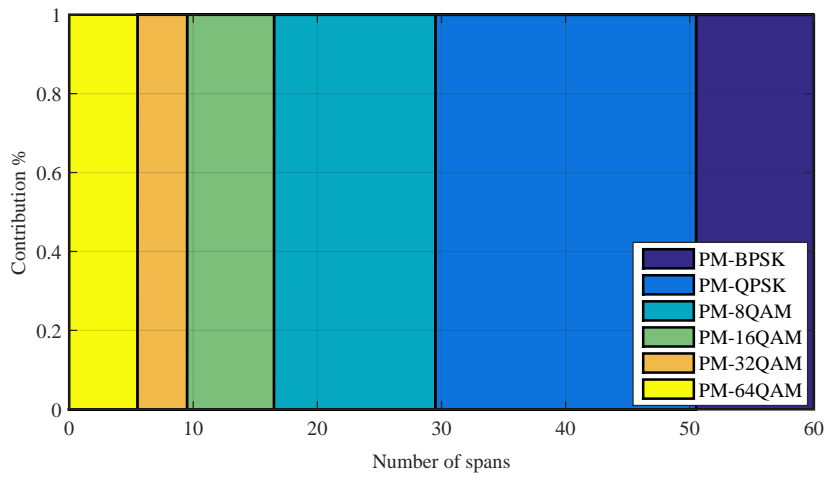

(a)

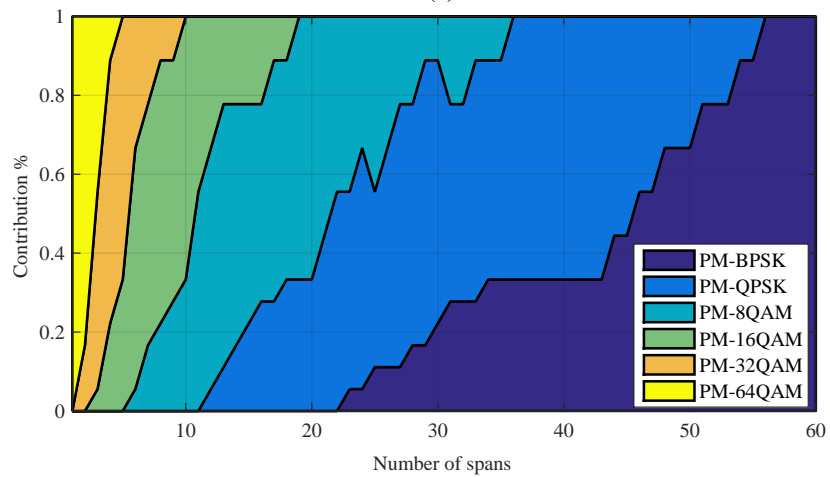

(b)

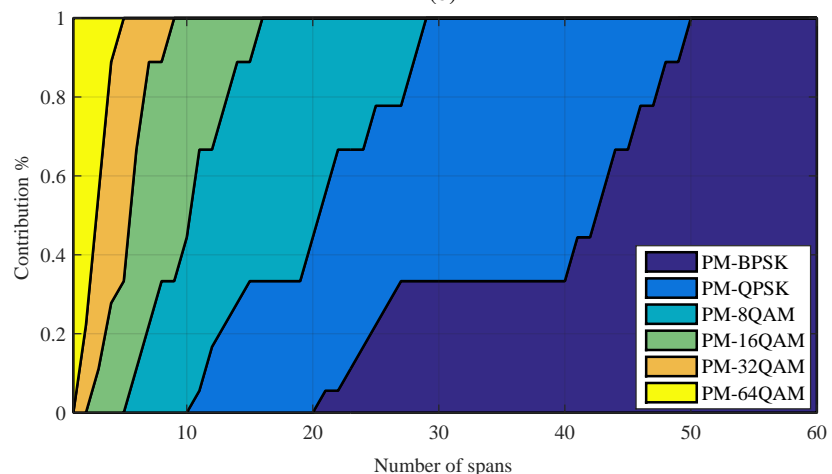

(c)

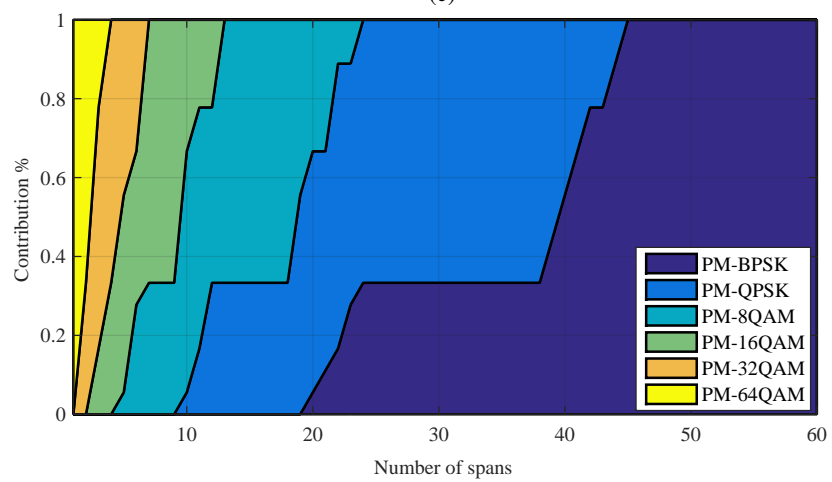

(d)

Figure 8. The contribution of each modulation format as function the transmission distance: (a) transmission reach method (b) 100 Gbps per channel (c) 200 Gbps per channel (d) 400 Gbps per channel.

nodes.

Figure 10 shows the running times of the benchmark and proposed algorithms, both of which grow exponentially with $|D|$, the number of integer variables in (8). The proposed algorithm uses slightly longer time than the benchmark due 


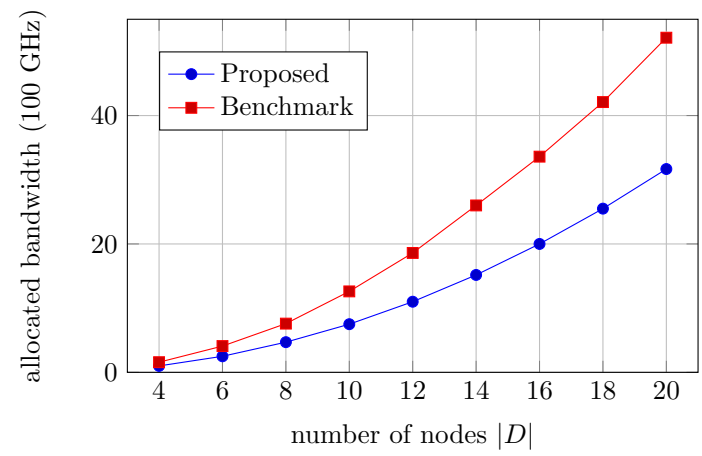

Figure 9. The bandwidth usages of the benchmark and proposed algorithms as a function of the number of nodes in ring networks.

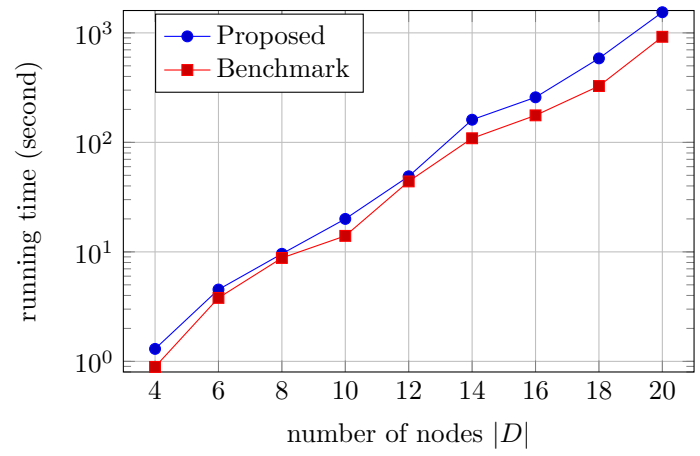

Figure 10. The running times of both algorithms as a function of the number of nodes.

to the flexible channel carrier frequencies, which require more computational effort to optimize than in fixed-grid networks. Note that the computational complexity of the proposed algorithm depends mainly on the number of integer variables, which equals to $|D|$ and does not change irrespective of the network topology. Therefore, the running time for meshed networks is similar to the ring networks.

The solution obtained for the ring networks with gridless spectrum is also extended to networks with finite grid size. A network with the central frequency granularity of 1.5625 $\mathrm{GHz}$ and a bandwidth granularity of $3.125 \mathrm{GHz}$ is used here. We first devide the spectrum into frequency slots of 3.125 $\mathrm{GHz}$, then the solution in gridless network is rounded to finite grid spectrum in such a way that each channel can occupy multiple frequency slots but one slot belongs to one channel at most [24]. This rounding scheme makes sure that the constraint (9) is always satisfied. Due to the fine grid granularity, the bandwidth usages increase slightly by only $2 \%$, which shows little impact of the finite-grid size on the proposed algorithm.

\section{CONCLUSIONS}

In this paper we presented a novel formulation to optimize the resource allocation in flexible-grid networks based on nonlinear signal distortion model. The resources allocated include bandwidths, carrier frequencies, modulation formats, and PSDs. By varying the weights of four different objectives, we can obtain a balance in minimizing the bandwidth usage, reducing the link nonlinearities, and maintaining robust SNR margins. With pre-determined routing and spectrum ordering, numerical results for a 3-node network indicate significant reductions in bandwidth and substantial increase in the overall throughput and transmission distance compared to a fixed-grid WDM benchmark. When the bandwidth usage minimization is prioritized, our proposed method is relatively insensitive to the channel ordering, which reduces the algorithm complexity considerably. Contrary to the well-known transmission reach method that assigns one fixed modulation format to a given distance, our proposed algorithm indicates that combining a range of modulation formats can produce closer to optimal solutions. The performance gains of the proposed algorithm crease significantly, while the complexity scales similarly as the benchmark.

\section{REFERENCES}

[1] R. Ramaswami, K. Sivarajan, and G. Sasaki, Optical Networks: A Practical Perspective. Morgan Kaufmann, 2009.

[2] O. Gerstel, M. Jinno, A. Lord, and S. Yoo, "Elastic optical networking: a new dawn for the optical layer?" IEEE Commun. Mag., vol. 50, no. 2, pp. s12-s20, February 2012.

[3] K. Christodoulopoulos, I. Tomkos, and E. Varvarigos, "Elastic bandwidth allocation in flexible OFDM-based optical networks," IEEE J. Lightw. Technol., vol. 29, no. 9, pp. 1354-1366, 2011.

[4] S. Talebi, F. Alam, I. Katib, M. Khamis, R. Salama, and G. N. Rouskas, "Spectrum management techniques for elastic optical networks: A survey," Opt. Switch. and Netw., vol. 13, pp. 34-48, 2014.

[5] A. Klekamp, R. Dischler, and F. Buchali, "Limits of spectral efficiency and transmission reach of optical-ofdm superchannels for adaptive networks," IEEE Photon. Technol. Lett., vol. 23, no. 20, pp. 1526-1528, 2011.

[6] P. Poggiolini, G. Bosco, A. Carena, V. Curri, Y. Jiang, and F. Forghieri, "The GN-model of fiber non-linear propagation and its applications," IEEE J. Lightw. Technol., vol. 32, no. 4, pp. 694-721, 2014.

[7] P. Johannisson and M. Karlsson, "Perturbation analysis of nonlinear propagation in a strongly dispersive optical communication system," IEEE J. Lightw. Technol., vol. 31, no. 8, pp. 1273-1282, 2013.

[8] P. Johannisson and E. Agrell, "Modeling of nonlinear signal distortion in fiber-optic networks," IEEE J. Lightw. Technol., vol. 32, no. 23, pp. 3942-3950, 2014.

[9] D. J. Ives and S. J. Savory, "Transmitter optimized optical networks," in Optical Fiber Communication Conference (OFC). Optical Society of America, 2013, pp. JW2A-64.

[10] D. J. Ives, P. Bayvel, and S. J. Savory, "Adapting transmitter power and modulation format to improve optical network performance utilizing the gaussian noise model of nonlinear impairments," IEEE J. Lightw. Technol., vol. 32, no. 21, pp. 3485-3494, 2014.

[11] S. J. Savory, "Congestion aware routing in nonlinear elastic optical networks," IEEE Photon. Technol. Lett., vol. 26, no. 10, pp. 1057-1060, 2014.

[12] E. Palkopoulou, G. Bosco, A. Carena, D. Klonidis, P. Poggiolini, and I. Tomkos, "Nyquist-WDM-based flexible optical networks: Exploring physical layer design parameters," IEEE J. Lightw. Technol., vol. 31, no. 14, pp. 2332-2339, 2013.

[13] O. Vassilieva, T. Yamauchi, S. Oda, I. Kim, T. Hoshida, Y. Aoki, J. C. Rasmussen, and M. Sekiya, "Systematic analysis of intra-superchannel nonlinear crosstalk in flexible grid networks," in European Conference on Optical Communication (ECOC), 2014.

[14] J. Zhao, H. Wymeersch, and E. Agrell, "Nonlinear impairment aware resource allocation in elastic optical networks," in Optical Fiber Communication Conference (OFC), 2015, p. M2I.1.

[15] L. Yan, E. Agrell, H. Wymeersch, P. Johannisson, R. Di Taranto, and M. Brandt-Pearce, "Link-level resource allocation for flexible-grid nonlinear fiber-optic communication systems," IEEE Photon. Technol. Lett., vol. 27, no. 12, pp. 1250-1253, June 2015.

[16] H. Beyranvand and J. A. Salehi, "A quality-of-transmission aware dynamic routing and spectrum assignment scheme for future elastic optical networks," IEEE J. Lightw. Technol., vol. 31, no. 18, pp. 30433054, 2013. 
[17] D. J. Ives, P. Bayvel, and S. J. Savory, "Physical layer transmitter and routing optimization to maximize the traffic throughput of a nonlinear optical mesh network," in IEEE International Conference of Optical Network Design and Modeling (ONDM), 2014.

[18] L. Yan, E. Agrell, and H. Wymeersch, "Resource allocation in nonlinear flexible-grid fiber-optic networks," in Optical Fiber Communication Conference (OFC), 2015, pp. Tu2I-5.

[19] P. Poggiolini, "The GN model of non-linear propagation in uncompensated coherent optical systems," IEEE J. Lightw. Technol., vol. 30, no. 24 pp. 3857-3879, 2012.

[20] N. Irukulapati, H. Wymeersch, P. Johannisson, and E. Agrell, "Stochastic digital backpropagation," IEEE Trans. Commun., vol. 62, no. 11, pp. 3956-3968, Nov 2014.

[21] P. Bonami et al., "An algorithmic framework for convex mixed integer nonlinear programs," Discret. Optim., vol. 5, no. 2, pp. 186-204, 2008.

[22] P. Bonami, M. Kilinç, and J. Linderoth, "Algorithms and software for convex mixed integer nonlinear programs," in Mixed Integer Nonlinear Programming. Springer, 2012, pp. 1-39.

[23] X. Wang, M. Brandt-Pearce, and S. Subramaniam, "Impact of wavelength and modulation conversion on transluscent elastic optical networks using milp," arXiv preprint arXiv:1412.8086, 2014.

[24] Spectral grids for WDM applications: DWDM frequency grid, Telecommunication Standardization Sector of International Telecommunication Union Recommendation ITU-T G.694.1, 2012. 